\title{
There and back again: Autoimmune Polyendocrinopathy Syndrome Type I and the Aire knockout mouse
}

\author{
Adrian Liston
}

Immunogenomics Laboratory, John Curtin School of Medical Research, The Australian National University, Canberra 260I, Australia

\begin{abstract}
Autoimmune Polyendocrinopathy Syndrome Type I (APSI) is a severe human multiorgan autoimmune disorder, caused by mutations in the gene AIRE. Following this determination, a mouse model for the disease was rationally designed by knocking out the murine homologue, Aire. The Aire knockout mouse has demonstrated that Aire initiates immune tolerance via promiscuous transcription of organ-specific genes in the thymus. Recent studies using the Aire knockout mouse have demonstrated gene-dose dependency and disease modifier loci, returning the onus of exploration to human genetics.
\end{abstract}

\section{Introduction - disease to gene}

Autoimmune Polyendocrinopathy Syndrome type 1 (APS1), also known as Autoimmune Polyendocrinopathy-Candidiasis-Ectodermal Dystrophy (APECED), is a severe disease characterised by autoimmunity against multiple peripheral organs (http://www.ncbi.nlm.nih.gov OMIM \#240300). APS1 is a rare condition, most commonly found in Finnish, Sardinian and Iranian Jewish populations. The most common manifestations are HYPOPARATHYROIDISM, primary adrenocortical failure and CHRONIC MUCOCUTANEOUS CANDIDIASIS and clinical diagnosis of APS1 requires the presence of at least two out of three of these primary disorders. There is considerable variation in the combination of symptoms apparent in each patient, with multiple additional disorders occurring

E-mail address: A. Liston (adrian.liston@anu.edu.au)

\section{Section Editor:}

Daniel Altmann - Human Disease Immunogenetics Group, Hammersmith Hospital, London, UK

in addition to the three primary disorders. APS1 was determined to be a recessive monogenic disorder, on the basis of a sibling penetrance of $24-25 \%$ [1]. The responsible allele was mapped to $21 \mathrm{q} 22.3$ [2], and subsequently determined to be loss-of-function mutations in the gene AIRE [3,4]. More than 50 nonsense and missense polymorphisms in AIRE are now known to be associated with APS1 [5].

\section{Disease models}

APS1 is a systems disease, requiring the complex interaction of immune cells with organs throughout the body. As such, no in vitro or in silico systems have been developed to model the disease state, although the in vitro studies on the AIRE protein have been of undoubted value in determining the biochemical activity of AIRE (see below). As a monogenic Mendelian disorder caused by loss of AIRE, in vivo models were able to be rationally designed by knocking out the murine homolog Aire (with 73\% sequence identity). Several separate Aire knockout mouse strains have been developed, of which the three published strains are summarised in Table 1. Although the knockout strains were produced in slightly different ways (as outlined in Table 1), the phenotype for each model appears essentially the same, thus there are no identified pros or cons to the different models. All independent strains have demonstrated spontaneous lymphocytic infiltration into multiple organs and the development of 


\section{Glossary}

Addison's disease: autoimmune-induced failure of the adrenal cortex with reduced production of adrenocorticotropic hormone. AIRE: autoimmune regulator.

APECED: Autoimmune Polyendocrinopathy-Candidiasis-Ectodermal Dystrophy

APS I: Autoimmune Polyendocrinopathy Syndrome Type I

CBP: CREB-binding protein

Chronic mucocutaneous candidiasis: persistent and/or recurrent infections of the skin, nails and mucous membranes with Candida species (mainly Candida albicans)

HEL: hen egg lysozyme

Hypoparathyroidism: autoimmune-induced failure of the parathyroid gland and reduced production of parathyroid hormone Idd: insulin-dependent diabetes loci (murine)

IDDM: insulin-dependent diabetes mellitus loci (human)

insHEL: insulin-promoter driven hen egg lysozyme

insOVA: insulin-promoter driven ovalbumin

Modifier loci: loci capable of shifting the clinical manifestations induced by autoimmune loci.

MTEC: medullary thymic epithelial cell.

OVA: ovalbumin.

Tolerogenic: induction of immunological nonreactivity to self.

autoantibodies. This spontaneous autoimmunity towards multiple organs parallels the human phenotype, albeit with far lower severity because this infiltration rarely progresses into clinical disease and never into any of the three primary characteristic disorders. This deviance from the human disease is of unknown origin, but might reflect the relatively resistant background of the C57BL/6 strain to autoimmunity (on which the knockout mice were originally characterised, see below); differential reliance upon Airedependent and Aire-independent tolerance mechanisms between humans and mice; or simply physiological differences in target organ resistance to autoimmunity. Regardless, studies using these in vivo models have led to dramatic increases in the knowledge of the mechanisms initiating autoimmunity in APS1 patients.

\section{Elucidation of the mechanism of Aire activity}

The tolerogenic role of Aire

Initial studies on Aire expression demonstrated that neither human nor murine AIRE/Aire is expressed in the target organs of autoimmunity, but rather is expressed in rare stromal cells in the thymic medulla and in dendritic cells in secondary lymphoid organs [6-8]. This finding led to the premise that AIRE functions through the induction of immunological tolerance of $\mathrm{T}$ cells. In particular, the intriguing possibility existed that Aire could be connected to the 'promiscuous' expression of organ-specific antigens in the thymic medulla, although few studies had previously linked this low level of expression to tolerance induction [9]. A key breakthrough in the field came through the analysis of Aire knockout thymi [10]. Through bone-marrow chimeric and thymus transplant studies it was demonstrated that the TOLEROGENIC role of Aire resides in the thymic stroma. Importantly, on sorted medullary thymic epithelial cells (mTECs), gene-chip analysis demonstrated the reduced expression of several organ-specific antigens, including potential target autoantigens for APS1 diseases, such as pro-insulin [10]. It has since been estimated that more than 2000-3000 organ-specific antigens are expressed in human and murine mTECs [11,12], of which $\sim 500$ might be Aire-dependent $[10,12]$. The connection of this transcriptional role for Aire to a tolerogenic mechanism came through the use of 3A9 TCR transgenic mice, whereby the majority of the $\mathrm{T}$ cell repertoire is reactive for the model antigen hen egg lysozyme (HEL). When the TCR transgenic mice are crossed to mice expressing HEL under the insulin promoter (insHEL), HEL expression in the thymic stroma directs the clonal deletion of autoreactive T cells [13]. In the absence of Aire, the thymic expression of HEL is ablated, leading to the defective clonal deletion of autoreactive $\mathrm{T}$ cells, the peripheral accumulation of autoreactive T cells and the development of autoimmune disease $[14,15]$. Thus, it appears that the tolerogenic role of Aire is to provide thymic expression of otherwise organ-specific antigens, allowing the clonal selection process to purge the autoreactive clones from the $\mathrm{T}$ cell repertoire (Fig. 1).

\section{The transcriptional role of Aire}

Currently, little is known about the mechanism by which Aire regulates the transcription of organ-specific genes in the thymic epithelium. In vitro studies of the biochemical properties of Aire, reviewed in [16], indicate that Aire might

Table I. In vitro models for APS I

\begin{tabular}{|c|c|c|c|}
\hline Group & Design & Autoimmune symptoms & Refs \\
\hline $\begin{array}{l}\text { Medical Genetics and Molecular Medicine, } \\
\text { University of Helsinki, Finland. }\end{array}$ & $\begin{array}{l}\text { Neo cassette insertion in exon } 6 \text {, analogous } \\
\text { to the most common human mutation. }\end{array}$ & $\begin{array}{l}\text { Lymphocytic infiltrate in ovaries, liver, adrenals. } \\
\text { Autoantibodies, reduced fertility. }\end{array}$ & {$[6]$} \\
\hline $\begin{array}{l}\text { Immunology and Immunogenetics, } \\
\text { Harvard Medical School, US. }\end{array}$ & $\begin{array}{l}\text { Cre-lox mediated deletion of exon } 2 \text { with } \\
\text { premature stop codon created in exon } 3 .\end{array}$ & $\begin{array}{l}\text { Lymphocytic infiltrate in salivary gland, retina, ovaries, } \\
\text { stomach, pancreas, liver, thyroid. Autoantibodies. }\end{array}$ & {$[10]$} \\
\hline $\begin{array}{l}\text { Molecular Immunology, } \\
\text { University of Tokushima, Japan. }\end{array}$ & $\begin{array}{l}\text { Neo cassette insertion replacing } \\
\text { exons } 5-12 \text {. }\end{array}$ & $\begin{array}{l}\text { Lymphocytic infiltrate of the lacrimal glands, } \\
\text { parotid glands, submandibular glands, stomach, } \\
\text { pancreas. Autoantibodies. }\end{array}$ & [27] \\
\hline
\end{tabular}




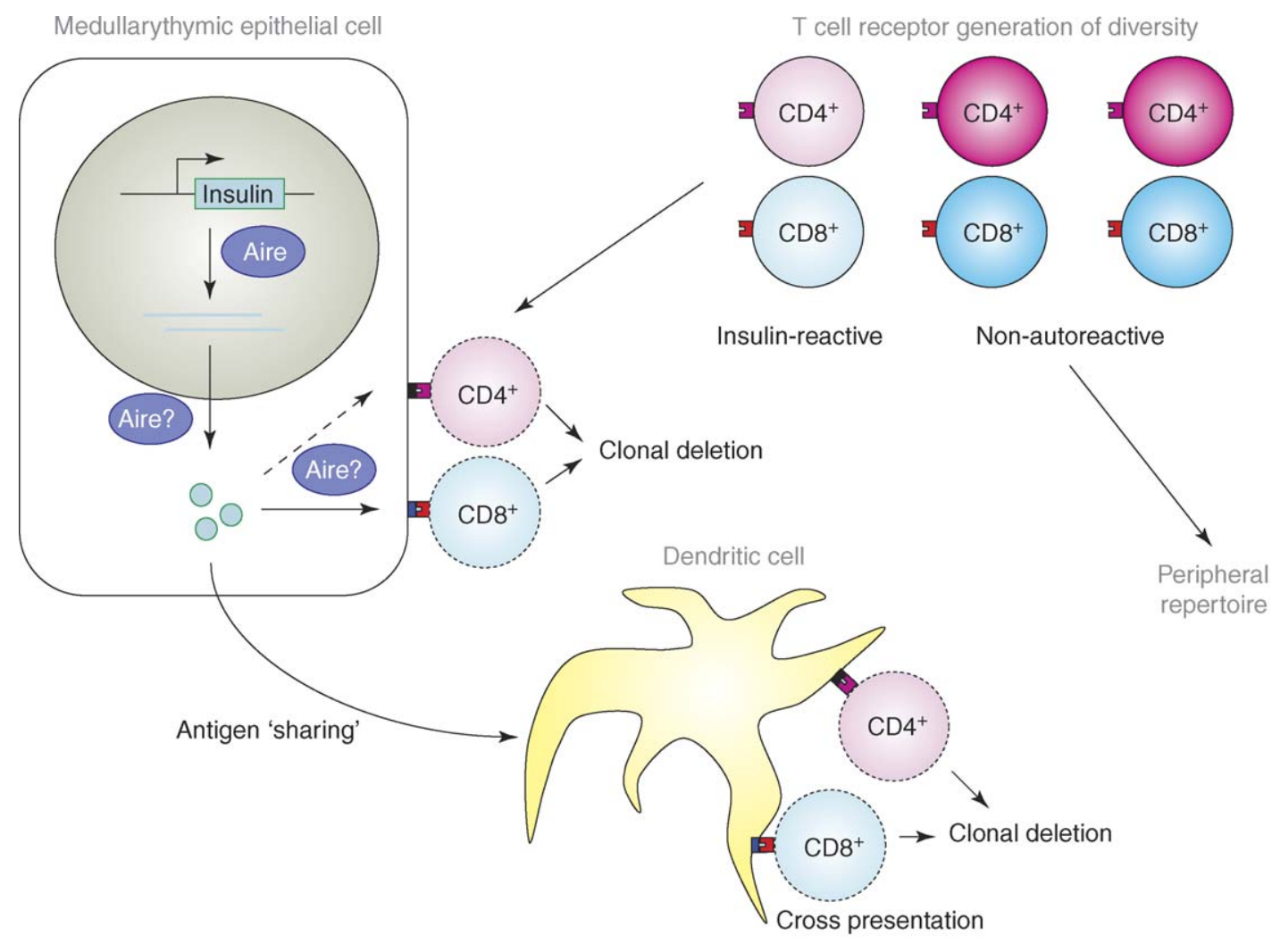

Drug Discovery Today: Disease Models

Figure I. Tolerogenic pathway of Aire. Generation of diversity in T cell receptors leads to the development of both autoreactive and non-autoreactive thymocytes. Expression of Aire in specialised epithelial cells in the thymic medulla (mTECs) leads to the transcription of organ-specific antigens, such as insulin, in an as-yet undetermined manner. Translation and processing of these antigens, which might also be affected by Aire, lead to presentation at the cell surface to thymocytes as a peptide/MHC complex. These complexes are able to induce directly the negative selection of MHC class I-restricted autoreactive thymocytes, and might also negatively select MHC class II-restricted autoreactive thymocytes. These antigens are also 'shared' with local resident dendritic cells, and following processing can induce deletion of both MHC class I- and class II-restricted autoreactive thymocytes. By purging the T cell repertoire of such autoreactive $\mathrm{T}$ cell clones in the thymus, Aire is able to prevent peripheral activation and autoimmunity.

act as a transcription factor, as it localises within the nuclear speckles [17,18] and interacts with CBP (transcriptional coactivator CREB-binding protein) [19]. Furthermore, homodimeric and homotetrameric Aire are capable of binding DNA [20], and when tethered to DNA, Aire is capable of activating transcription $[19,21]$. There are, however, several lines of evidence that Aire does not act in the manner of a classical transcription factor. First, the scale of promiscuous antigen expression, at an estimated 500 genes $[10,12]$, implicates a nondirect role in gene transcription. Second, the clustering of Aire-dependent transcripts might enable transcriptional control via higher order chromatin regulation [12,22,23]. Third, the E3 ubiquitin-ligase activity of the Aire protein suggests a nontraditional function in increasing promoter activity [24], although this has been disputed [25]. One such nonclassical transcriptional model has been a proposed function for Aire as a higher order chromatin remodelling protein, opening up chromatin structure in the clusters of organ-specific genes. An alternative model, proposed by Farr et al. [26], is based on the intriguing finding that lung epithelial transcripts localise to thymic cysts with ultrastructural properties resembling lung epithelium. In this model it is proposed that rather than directing the transcription of each organ-specific transcript, Aire instead directs the differentiation of clusters of thymic epithelium into specialised lineages, which then proceed to initiate organ-specific transcription in a classical manner. Further experiments will, however, be required to test if either of these attractive models is correct (see Outstanding issues).

\section{A potential antigen processing role of Aire}

Recently, several lines of data have come out that challenge the new model of Aire acting via thymic transcription of organ-specific antigens to induce central tolerance. In two cases a failure of tolerance has been observed in Aire knockout mice towards antigens that are not dependent on Aire for their thymic expression. In the first case, Aire knockout mice were found to have a strong anti- $\alpha$-fodrin autoimmune 
response with unchanged thymic $\alpha$-fodrin expression [27]. As an isolated datum this does not necessitate the inclusion of a second tolerogenic mechanism for Aire because it is unclear whether $\alpha$-fodrin is a primary immune target in this situation, or autoantibodies simply result from a breach in immunological ignorance caused by an autoimmune response against an alternative, Aire-dependent, autoantigen. The second line of evidence, however, is more perplexing because the use of a monoclonal repertoire removes this caveat. In this report, tolerance towards insulin promoter-driven ova (insOVA) is analysed in Aire knockout mice [28]. Like the insHEL model [14,15], loss of Aire results in defective negative selection of OVA-reactive T cells (both MHC class I-restricted OT-I transgenics and MHC class II-restricted OT-II transgenics) and development of autoimmune diabetes [28]. However, unlike insHEL and the endogenous insulin gene [10,14], insOVA expression in the thymus is unchanged in the Aire knockout [28].

These data suggest a role for Aire in thymic presentation of antigen, above and beyond the currently acknowledged role in thymic expression of antigen. This proposed expansion in the scope of Aire activity is perplexing owing to two different lines of evidence. First, the deletion of OT-II TCR transgenic thymocytes by the insOVA transgene has been elegantly demonstrated to be mediated by epithelial expression of OVA followed by thymic dendritic cell capture of unprocessed antigen and presentation to thymocytes [29]. Because the epithelial cells in the OT-II/insOVA system are not responsible for any biologically relevant levels of OVA processing or presentation, it is difficult to see how any role of Aire in these processes would disturb deletion of autoreactive thymocytes. Second, it is intriguing as to how a presumably cis-acting processing/presentation defect would affect tolerance towards OVA if the antigen was not restricted to Aireexpressing mTECs. Negative selection towards ubiquitous antigens, such as the male antigen [28] or HEL driven by the MHC class I promoter [14], and Aire-independent ectopic transcripts, such as human $\mathrm{C}$ reactive protein [28], is unaffected in Aire knockout mice. Thus, for antigens not restricted to Aire-expressing cells, the putative processing role of Aire does not effect negative deletion. Because it appears that insOVA does not require Aire to be transcribed in the thymus, it becomes difficult to formulate a mechanism by which insOVA is restricted to Aire-expressing mTECs, such that the processing role of Aire is limiting during negative selection. Even if these qualifications make a role for Aire in antigen processing or presentation unlikely, the question remains - why is tolerance towards insOVA Airedependent if transcription of insOVA is Aire-independent? Determination of the exact mechanism by which Aire is required for insOVA tolerance will be crucial for understanding the multiple layers of Aire tolerance mechanisms (see Outstanding issues).

\section{Model translation to humans}

Being a rationally designed in vivo model, with a homologous genetic lesion and similar pathologies to the human disease, the general tolerogenic role of Aire determined in Aire knockout mice is expected to be directly translatable to the tolerogenic role of AIRE in humans. As a model of the specific clinical condition of APS1, however, the Aire knockout mouse is unable to reproduce the combination of autoimmune diseases characteristic of APS1. Thus, the development of a high incidence of hypoparathyroidism, primary adrenocortical failure and candidiasis infection, the three classical symptoms of APS1, does not occur in Aire knockout mice. The simplest explanation for this discrepancy is that Aire knockout mice replicate the same fundamental defect in immunological tolerance, but the clinical manifestation is redirected towards alternative targets. In the case of candidiasis infection, the propensity for Candida to infect areas of subclinical damage might place the ultimate causation of candidiasis in APS1 patients on mucocutaneous autoimmunity. Thus, the origin of the deviance from APS1 might simply be an expansion of the clinical diversity in APS1, created by the increased influence of MODIFIER LOCI in interspecies comparisons. The dramatic influence of murine strain background on autoimmunity (both in severity and target, see below) in Aire knockout mice demonstrates the potential of modifier loci. The limited use of Aire knockout mice in modelling the specific clinical conditions associated with APS1 is therefore not a cause of concern for its fidelity to the tolerance mechanisms failing in both the mouse and the human. Beyond the determination of these mechanisms, the genetic interactions apparent in the Aire knockout mouse provide a potential translation into the human genetics of APS1 (see Outstanding issues).

\section{Sensitivity of the Aire pathway to subtle modification}

One surprising outcome of the studies on the Aire knockout mice has been the exquisite sensitivity that the Aire pathway shows to the loss of a single copy of Aire. Aire heterozygote mice show the same number of Aire-expressing cells in the thymic medulla, however each cell expresses less Aire [14]. As a result, the expression of both endogenous insulin and insHEL is reduced two- to threefold. Using the HEL-reactive TCR transgenic $\times$ insHEL transgenic strain described above, this partial reduction in insHEL expression in Aire heterozygotes results in the increased escape of autoreactive HELresponsive thymocytes to the periphery, and an increase in the incidence of autoimmune disease [14]. A similar finding has been observed in the insOVA system, where Aire heterozygosity was linked to reduced clonal deletion and increased autoimmunity [28].

It is currently unknown if this gene-dose dependent effect is relevant to human disease. The sibling penetrance of 
24-25\% for APS1 indicates a strictly recessive trait, however this study was performed using the strict clinical criteria for APS1 (two of the three primary disorders). The association of the IDDM2 insulin promoter polymorphism with autoimmune diabetes in humans indicates that a two- to threefold reduction in thymic insulin levels (roughly equivalent to that observed with Aire heterozygosity in mice) is sufficient to predispose towards autoimmunity [30,31]. Thus,

(a)
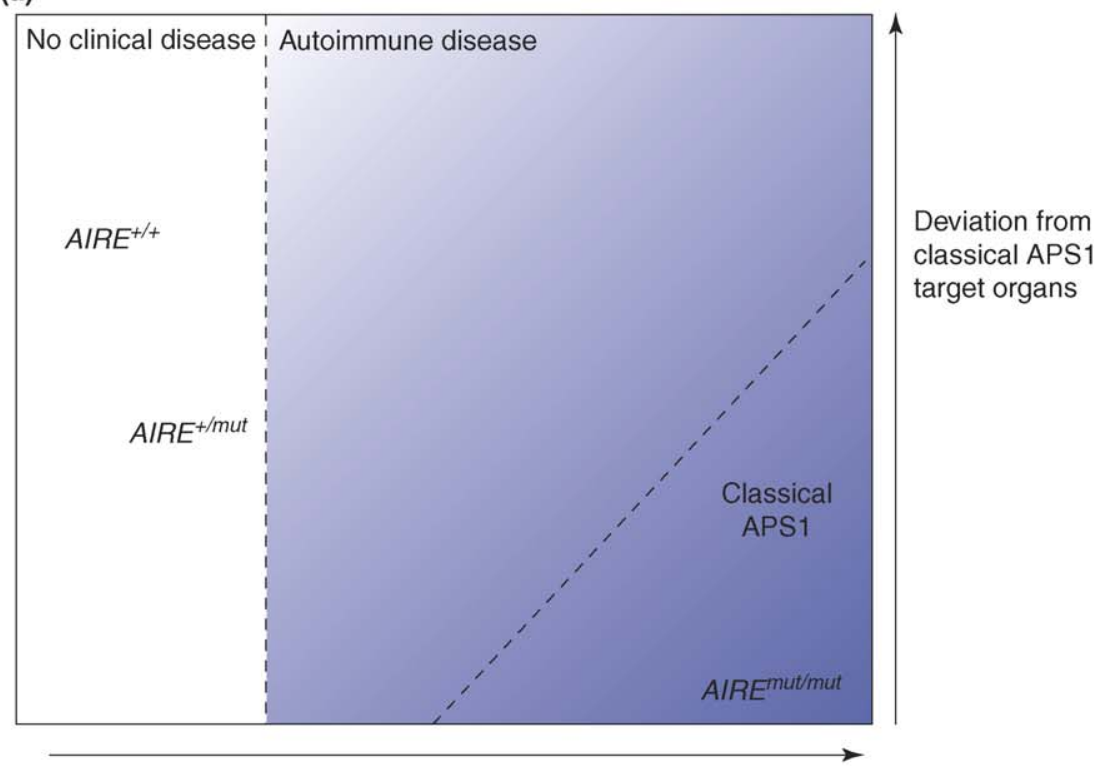

(b)

Number of organs affected

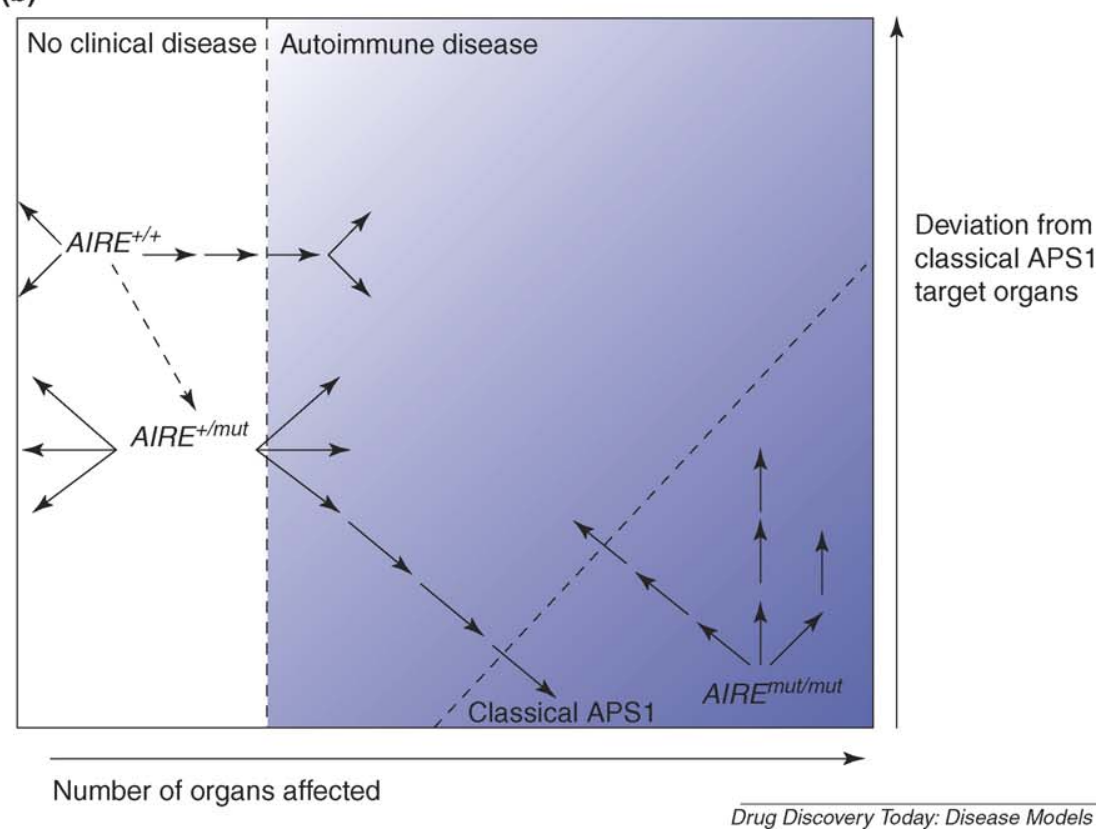

Figure 2. Clinical heat-map for genetic interactions in APSI. Clinical autoimmunity can be represented on a heat-map, representing the number of organs affected, and the deviation in target organ from classical APSI. The heat-map can be divided into three regions - that of no clinical disease (white), that of autoimmune disease (light blue) and that of classical APSI (dark blue). (a) In the unmodified situation, patients with no defects in AIRE, or a defect in only a single copy of AIRE, develop no clinical disease, whereas patients with homozygous AIRE defects develop classical APSI. (b) Layering the added complexity of AIRE gene-dose dependency and modifier loci recapitulates the diversity of clinical conditions observed. The effect of each modifier locus is represented by an arrow, indicating the clinical condition to which the modifier locus drives the basal state. Patients with homozygous AIRE defects are thus driven to increased deviation from the classical APSI clinical condition by modifier loci. The fortuitous combination of multiple resistance modifier loci can even in rare cases push the patient outside a classical APSI condition. Patients with heterozygous AIRE defects, although normally asymptomatic, can be driven to autoimmunity by modifier loci, even in extreme cases to a classical APSI condition. The development of common autoimmune diseases occurs in patients without AIRE mutations by multiple genetic interactions pushing the basal state towards autoimmunity. Note that AIRE heterozygosity can be represented as a modifier locus from the wild-type state (dashed arrow), insufficient to cause autoimmunity but with a cumulative effect. 
Table 2. Population frequency of AIRE mutations

\begin{tabular}{llll}
\hline Population & APSI frequency & Carrier frequency (\%) & Refs \\
\hline Finnish & $\mathrm{I} / 25,000$ & 1.3 & {$[40]$} \\
\hline Iranian Jews & $\mathrm{I} / 6500-1 / 9000$ & $2.1-2.5$ & {$[4 \mathrm{I}]$} \\
\hline Sardinian & $\mathrm{I} / 14,500$ & 1.7 & {$[42]$} \\
\hline
\end{tabular}

although it is unlikely that AIRE heterozygosity could lead to APS1 development, it is possible that mutation of a single copy of AIRE might act in a polygenic manner to contribute towards the development of common autoimmune diseases (Fig. 2). The large number of AIRE-dependent autoantigens means that a diverse set of autoimmune diseases could be influenced by AIRE heterozygosity. Currently this hypothesis has not been tested in a population with a significant frequency of AIRE heterozygosity, such as the Finnish, Sardinian or Iranian Jewish populations (Table 2). However, there is an anecdotal support for the hypothesis, with several APS1 patients that have had only a single AIRE mutation identified [21,32,33], and AIRE heterozygous patients with 'atypical' (less severe) APS1 [34,35]. Furthermore, a genedose dependent effect might synergise with the partial dominant negative behaviour of some APS1-causing AIRE alleles [36].

\section{Disease modifier loci in APSI}

One of the continuing clinical mysteries in APS1 has been the heterogeneity of target organ disease among patients, especially between different cohorts. For example, Iranian Jewish patients have less common candidiasis and ADDISON's DISEASE, whereas Finnish patients have more common diabetes mellitus [5]. Use of the Aire knockout mouse models of APS1 has recently suggested the origin of this variability. In two separate studies, Aire knockout mice were backcrossed to several different mouse strains, and the strain genetic background was found to significantly modify the disease symptoms. In the first study, the BALB/c background was shown to increase the incidence of gastric lymphocytic infiltrate and autoantibody production in Aire knockout mice, when compared with C57BL/6 Aire knockout mice [27]. In the second study, four different genetic backgrounds were used, C57BL/ $6, \mathrm{BALB} / \mathrm{c}, \mathrm{NOD}$ and SJL/J, and a distinct but overlapping set of pathologies developed between the different strains, with, for example, the development of the stomach as a target organ on the NOD and BALB/c backgrounds, and the exocrine pancreas tissue as a target on the NOD and SJL/J backgrounds [37]. The NOD genetic background was detrimental enough to turn the relatively mild autoimmune symptoms observed on the C57BL/ 6 background into a disease fatal by 14 weeks of age [37]. The disease modifier loci for pancreatitis mapped to known autoimmunity loci for insulin-dependent diabetes, Idd1 (the MHC locus), Idd3 and Idd5 [37]. These results implicate the existence of disease modifier loci in the human population, modifying the symptom set which develops in each patient (Fig. 2). Owing to the low numbers of APS1 patients available for linkage studies, modifier loci have not been identified (bar a putative influence of HLA $[33,38])$, however by extension from the NOD analysis, it might be the loci that are involved in the polygenic development of common autoimmune diseases that are also responsible for the disease modification in APS1 patients. Furthermore, the presence of rare individuals harbouring homozygous AIRE-null mutations [39] without developing APS1 might reflect a fortuitous constellation of autoimmune resistance modifier loci.

\section{Conclusions}

Determination of the genetic basis of APS1 allowed the rational design of a murine mouse model, the Aire knockout mouse. As a tool to dissect the point of immunological tolerance failure in the human disease, the Aire knockout mouse has been indispensable. Use of the knockout has resulted in rapid elucidation of the key tolerogenic mechanisms of Aire, with direct translation to APS1. This has allowed the development of the current model by which Aire initiates thymic transcription of organ-specific antigens, allowing clonal deletion mechanisms to purge the $\mathrm{T}$ cell repertoire of autoreactive clones. It has also provided key insights on the mechanisms by which tolerance might be broken in common autoimmune diseases, beyond the rare condition of APS1. Finally, the genetic interactions observed in the Aire knockout mouse have allowed the development of testable hypotheses for the contribution of human AIRE mutations to common autoimmune diseases, and the effect of modifier loci in producing the variability of the clinical condition.

\begin{tabular}{|ll|}
\hline Genbank accession numbers & \\
\hline Homo sapiens AIRE & 326 \\
Homo sapiens insulin & 3630 \\
Homo sapiens CBP & 1387 \\
Mus musculus Aire & 11634 \\
Mus musculus insulin 1 & 16333 \\
Mus musculus insulin 2 & 16334 \\
Mus musculus CBP & 12914 \\
Mus musculus $\alpha$-fodrin & 20740 \\
\hline
\end{tabular}

Links

- Aire knockout strains on Jax http://www.informatics.jax.org/ searches/ allele_report.cgi?_Marker_key=40880\&int:_Set_key $=847$ I 56

- APSI clinical data on OMIM http://www.ncbi.nlm.nih.gov/entrez/ dispomim.cgi?id=240300 


\section{Related articles}

Ramsey, C. et al. (2002) Aire deficient mice develop multiple features of APECED phenotype and show altered immune response. Hum. Mol. Genet. II, 397-409

Anderson, M.S. et al. (2002) Projection of an immunological selfshadow within the thymus by the aire protein. Science 298, I395-140I Liston, A. et al. (2003) Aire regulates negative selection of organspecific T cells. Nat. Immunol. 4, 350-354

Liston, A. et al. (2004) Gene dosage limiting role of Aire in thymic expression, clonal deletion and organ-specific autoimmunity. J. Exp. Med. 200, 1015-1026

Jiang, W. et al. (2005) Modifier loci condition autoimmunity provoked by Aire deficiency. J. Exp. Med. 202, 805-815

\section{Outstanding issues}

- Mechanism of transcriptional activation of organ-specific genes.

- Dissection of nontranscriptional activity of Aire.

- Connection between AIRE heterozygosity and autoimmune susceptibility in humans.

- Identification of modifier loci in altered disease symptoms in humans.

\section{Acknowledgements}

Stimulating discussion and manuscript review by Chris Goodnow and Lydia Makaroff were greatly appreciated. This work was supported by grants from the National Health and Medical Research Council, the Juvenile Diabetes Research Foundation and the EURAPS consortium.

\section{References}

1 Ahonen, P. (1985) Autoimmune polyendocrinopathy-candidosisectodermal dystrophy (APECED): autosomal recessive inheritance. Clin. Genet. 27, 535-542

2 Aaltonen, J. et al. (1994) An autosomal locus causing autoimmune disease: autoimmune polyglandular disease type I assigned to chromosome 21 . Nat. Genet. 8, 83-87

3 Nagamine, K. et al. (1997) Positional cloning of the APECED gene. Nat. Genet. 17, 393-398

4 The Finnish-German APECED Consortium, (1997) An autoimmune disease, APECED, caused by mutations in a novel gene featuring two PHDtype zinc-finger domains. Nat. Genet. 17, 399-403

5 Vogel, A. et al. (2002) The genetic background of autoimmune polyendocrinopathy-candidiasis-ectodermal dystrophy and its autoimmune disease components. J. Mol. Med. 80, 201-211

6 Ramsey, C. et al. (2002) Aire deficient mice develop multiple features of APECED phenotype and show altered immune response. Hum. Mol. Genet. 11, 397-409

7 Heino, M. et al. (1999) Autoimmune regulator is expressed in the cells regulating immune tolerance in thymus medulla. Biochem. Biophys. Res. Commun. 257, 821-825

8 Kogawa, K. et al. (2002) Expression of AIRE gene in peripheral monocyte/ dendritic cell lineage. Immunol. Lett. 80, 195-198

9 Hanahan, D. (1998) Peripheral-antigen-expressing cells in thymic medulla: factors in self-tolerance and autoimmunity. Curr. Opin. Immunol. $10,656-662$

10 Anderson, M.S. et al. (2002) Projection of an immunological self-shadow within the thymus by the Aire protein. Science 298, 1395-1401
11 Gotter, J. et al. (2004) Medullary epithelial cells of the human thymus express a highly diverse selection of tissue-specific genes colocalized in chromosomal clusters. J. Exp. Med. 199, 155-166

12 Derbinski, J. et al. (2005) Promiscuous gene expression in thymic epithelial cells is regulated at multiple levels. J. Exp. Med. 202, 33-45

13 Liston, A. et al. (2005) Genetic lesions in T-cell tolerance and thresholds for autoimmunity. Immunol. Rev. 204, 87-101

14 Liston, A. et al. (2004) Gene dosage limiting role of Aire in thymic expression, clonal deletion and organ-specific autoimmunity. J. Exp. Med. 200, 1015-1026

15 Liston, A. et al. (2003) Aire regulates negative selection of organ-specific T cells. Nat. Immunol. 4, 350-354

16 Villasenor, J. et al. (2005) AIRE and APECED: molecular insights into an autoimmune disease. Immunol. Rev. 204, 156-164

17 Heino, M. et al. (2000) RNA and protein expression of the murine autoimmune regulator gene (Aire) in normal, RelB-deficient and in NOD mouse. Eur. J. Immunol. 30, 1884-1893

18 Rinderle, C. et al. (1999) AIRE encodes a nuclear protein co-localizing with cytoskeletal filaments: altered sub-cellular distribution of mutants lacking the PHD zinc fingers. Hum. Mol. Genet. 8, 277-290

19 Pitkanen, J. et al. (2000) The autoimmune regulator protein has transcriptional transactivating properties and interacts with the common coactivator CREB-binding protein. J. Biol. Chem. 275, 16802-16809

20 Kumar, P.G. et al. (2001) The autoimmune regulator (AIRE) is a DNA-binding protein. J. Biol. Chem. 276, 41357-41364

21 Bjorses, P. et al. (2000) Mutations in the AIRE gene: effects on subcellular location and transactivation function of the autoimmune polyendocrinopathy-candidiasis-ectodermal dystrophy protein. Am.J. Hum. Genet. 66, 378-392

22 Johnnidis, J.B. et al. (2005) Chromosomal clustering of genes controlled by the aire transcription factor. Proc. Natl. Acad. Sci. U S A 102, 7233-7238

23 Tao, Y. et al. (2006) AIRE recruits multiple transcriptional components to specific genomic regions through tethering to nuclear matrix. Mol. Immunol. 43, 335-345

24 Uchida, D. et al. (2004) AIRE functions as an E3 ubiquitin ligase. J. Exp. Med. 199, 167-172

25 Bottomley, M.J. et al. (2005) NMR structure of the first PHD finger of autoimmune regulator protein (AIRE1). Insights into autoimmune polyendocrinopathy-candidiasis-ectodermal dystrophy (APECED) disease. J. Biol. Chem. 280, 11505-11512

26 Farr, A.G. et al. (2002) Organization of thymic medullary epithelial heterogeneity: implications for mechanisms of epithelial differentiation. Immunol. Rev. 189, 20-27

27 Kuroda, N. et al. (2005) Development of autoimmunity against transcriptionally unrepressed target antigen in the thymus of Airedeficient mice. J. Immunol. 174, 1862-1870

28 Anderson, M.S. et al. (2005) The cellular mechanism of Aire control of T cell tolerance. Immunity 23, 227-239

29 Gallegos, A.M. and Bevan, M.J. (2004) Central tolerance to tissue-specific antigens mediated by direct and indirect antigen presentation. J. Exp. Med. 200, 1039-1049

30 Vafiadis, P. et al. (1997) Insulin expression in human thymus is modulated by INS VNTR alleles at the IDDM2 locus. Nat. Genet. 15, 289-292

31 Pugliese, A. etal. (1997) The insulin gene is transcribed in the human thymus and transcription levels correlated with allelic variation at the INS VNTRIDDM2 susceptibility locus for type 1 diabetes. Nat. Genet. 15, 293-297

32 Wang, C.Y. et al. (1998) Characterization of mutations in patients with autoimmune polyglandular syndrome type 1 (APS1). Hum. Genet. 103, $681-685$

33 Halonen, M. et al. (2002) AIRE mutations and human leukocyte antigen genotypes as determinants of the autoimmune polyendocrinopathycandidiasis-ectodermal dystrophy phenotype. J. Clin. Endocrinol. Metab. $87,2568-2574$

34 Buzi, F. et al. (2003) Autoimmune polyendocrinopathy-candidiasisectodermal dystrophy syndrome: time to review diagnostic criteria? J. Clin. Endocrinol. Metab. 88, 3146-3148

35 Cetani, F. et al. (2001) A novel mutation of the autoimmune regulator gene in an Italian kindred with autoimmune polyendocrinopathy-candidiasis- 
ectodermal dystrophy, acting in a dominant fashion and strongly cosegregating with hypothyroid autoimmune thyroiditis. J. Clin.

Endocrinol. Metab. 86, 4747-4752

36 Ilmarinen, T. et al. (2005) Functional analysis of SAND mutations in AIRE supports dominant inheritance of the G228W mutation. Hum. Mutat. 26, 322-331

37 Jiang, W. et al. (2005) Modifier loci condition autoimmunity provoked by Aire deficiency. J. Exp. Med. 202, 805-815

38 Kogawa, K. et al. (2002) Distinct clinical phenotype and immunoreactivity in Japanese siblings with autoimmune polyglandular syndrome type 1 (APS-1) associated with compound heterozygous novel AIRE gene mutations. Clin. Immunol. 103 (3 Pt. 1), 277-283
39 Boe, A.S. et al. (2002) Mutational analysis of the autoimmune regulator (AIRE) gene in sporadic autoimmune Addison's disease can reveal patients with unidentified autoimmune polyendocrine syndrome type I. Eur. J. Endocrinol. 146, 519-522

40 Ahonen, P. et al. (1990) Clinical variation of autoimmune polyendocrinopathy-candidiasis-ectodermal dystrophy (APECED) in a series of 68 patients. N. Engl. J. Med. 322, 1829-1836

41 Zlotogora, J. and Shapiro, M.S. (1992) Polyglandular autoimmune syndrome type I among Iranian Jews. J. Med. Genet. 29, 824-826

42 Rosatelli, M.C. et al. (1998) A common mutation in Sardinian autoimmune polyendocrinopathy-candidiasis-ectodermal dystrophy patients. Hum. Genet. 103, 428-434 